\title{
Estudo preliminar do depósito de paligorsquite de Figueiró do Campo (Soure, Portugal)
}

\section{Preliminary studies of the palygorskite deposit from Figueiró do Campo (Soure, Portugal)}

\author{
MOURA, A. ${ }^{1}$, LOPES VELHO, J. ${ }^{2}$ e ALVES, W. ${ }^{3}$
}

(1) Departamento de Geociências, Ambiente e Ordenamento do Território. Faculdade de Ciências da Universidade do Porto. Rua do Campo Alegre 4167-007 Porto, Portugal. Centro de Geologia da Universidade do Porto. Email: ajmoura@fc.up.pt

(2) Departamento de Geociências. Universidade de Aveiro. Campo de Santiago, 3810-146 Aveiro, Portugal.

(3) Mestrando no Departamento de Geociências da Universidade de Aveiro. Campo de Santiago, 3810146 Aveiro, Portugal

https://doi.org/10.17979/cadlaxe.2015.38.0.3685

\begin{abstract}
The technical characteristics of miocenic palygorskite rich-sediments from an area near Figueiró do Campo (Soure, Portugal) are presented. The deposit is probably the most promising area in Portugal concerning palygorskite as a resource. In this first study we evaluate three samples collected in the bottom, medium and top levels of the deposit. The samples has 58-79\% in weight under $63 \mu$ and the average particle size in the fraction $<63 \mu$ has 0.2 to $2.2 \mu$ in size. DRX data revealed that the fraction $<63 \mu$ are 26-34 \% quartz, 4-6\% Fe oxide and 61-70\% palygorskite. The fraction $<2 \mu$ is $89-93 \%$ palygorskite, $4-8 \%$ quartz, $3-4 \%$ Fe oxide and $0-3 \%$ caolinite. The palygorskite fibbers were observed in MEV and average $344 \mathrm{~nm}$ (length) and $31 \mathrm{~nm}$ (thickness). From FRX analysis we obtain the following compositions: $69-60 \% \mathrm{SiO}_{2}, 11-17 \% \mathrm{Al}_{2} \mathrm{O}_{3}, 7.5-5.1$ $\% \mathrm{MgO}$ and 3.6-6.6 \% Fe total. CTC are in the range 7.2-11.2 meq/100g, and the $\mathrm{pH}$ between 6.0
\end{abstract}


and 7.1. The moisture are between 9.21 and $15.08 \%$. Oil and water sorption are respectively in the range $69-100 \%$ and $75-134 \%$. Einlehner abrasivity is between 11 and $80 \mathrm{mg}$ in the fraction $<63$ $\mu$, and $1.2-1.9 \mathrm{mg}$ in the fraction $<2 \mu$.

In the overall results do not exclude the possibility of a feasible exploitation. It is thus essential to proceed with a geophysical study (to determine the thickness and form of the deposit) and with boreholes exploration in order to get fresh samples from the interior of the mineralized level.

The deposit was probably formed in a restricted basin filled with alkaline waters and the palygorskite precipitated from solutions satured with silica and magnesium although we cannot preclude a formation after an esmectite precursor.

Key words: palygorskite; deposit; Portugal 


\section{INTRODUÇÃO}

A paligorsquite (também conhecida como atapulgite) é uma argila fibrosa com grande interesse industrial, sendo utilizada em aplicações tão diversas como a indústria farmacêutica e de cosmética, em camas para animais, em fluidos de sondagem, em fertilizantes, em pesticidas, na clarificação de vinhos, como absorvente industrial, entre outras (HEIVILIN, 1994).

Existem em Espanha várias explorações de paligorsquite. Na região de Cáceres situa-se aquele que tem sido considerado o maior jazigo europeu desta argila. Em Portugal, no entanto, a argila é mal conhecida, nunca tendo sido explorada. Porém, encontram-se referenciadas rochas sedimentares com paligorsquite em vários pontos de Portugal, todos em litologias do Cenozoico. O estudo de rochas sedimentares portuguesas ricas em paligorsquite foi pela primeira vez efetuado por GALOPIM DE CARVALHO (1968). Este geólogo estudou ambas as margens da bacia do rio Tejo identificando a argila em vários locais, embora em nenhum deles ocorresse em quantidade que fizesse prever o seu aproveitamento industrial. Posteriormente, REIS (1983) e CUNHA E REIS (1989) descreveram alguns locais, na bacia Lusitaniana, com paligorsquite na fração argilosa. DIAS (1994) dedicou a sua tese de doutoramento ao estudo de formações ricas em paligorsquite tendo estudado formações das bacias do Tejo, Douro e Guadiana, onde encontrou três locais com potencial interesse económico.

O objetivo do presente trabalho é contribuir para um melhor conhecimento da paligorsquite em Portugal através do estudo do depósito de Figueiró do Campo, localizado a cerca de $30 \mathrm{~km}$ a SW de Coimbra, na região centro de Portugal pertencente à bacia do rio Mondego. O trabalho versou, principalmente, a determinação de um conjunto de características mineralógicas, químicas e tecnológicas de amostras do nível rico em paligorsquite. Serviu ainda para que se tecessem algumas ilações relativas à génese do depósito.

Este trabalho enquadra-se num projecto mais vasto de caracterização da paligorsquite em Portugal.

\section{GEOLOGIA REGIONAL E LOCAL}

O litótipo objeto do presente estudo pertence aos depósitos continentais da Formação dos Grés e Argilas de Amor (Miocénico médio) que fazem parte da Bacia Lusitaniana da Orla meso-cenozoica de Portugal e se distribuem numa área de algumas centenas de quilómetros quadrados entre os paralelos de Nazaré e de Coimbra (CUNHA E REIS, 1992). Segundo PAIS et al. (2010) esta formação constitui uma unidade alostratigráfica (sequência limitada por descontinuidades sedimentares) da designada Bacia do Mondego. A formação foi estudada por vários autores e, em particular, por REIS (1983) que a considerou como provável equivalente lateral do membro III da Formação do Bom Sucesso. Este autor descreveu no sector Condeixa-Alfarelos a área de Figueiró do Campo (concelho de Soure) onde afloram depósitos ricos em paligorsquite. Um destes depósitos foi alvo do interesse de uma empresa privada nos anos 1989-90, tendo em vista um eventual aproveitamento do depósito.

O depósito abrange uma área de aproximadamente $165000 \mathrm{~m}^{2} \mathrm{e}$ localiza-se a WSW da aldeia de Figueiró do Campo situando-se na folha 19-C da Carta Geológica de Portu- 
gal à escala 1:50 000 (figura 1). O nível é praticamente horizontal tendo na região uma espessura que varia entre 5 e $25 \mathrm{~m}$. A muro encontra-se limitado por um nível conglomerático bastante silicificado e a topo é limitado por um depósito detrítico de cobertura
(Pliocénico) com espessura igual ou inferior a $10 \mathrm{~m}$. A espessura média do nível rico em paligorsquite, difícil de estimar, deverá rondar os $10 \mathrm{~m}$. A algumas centenas de metros do depósito ocorrem outros, análogos, embora de menor área.

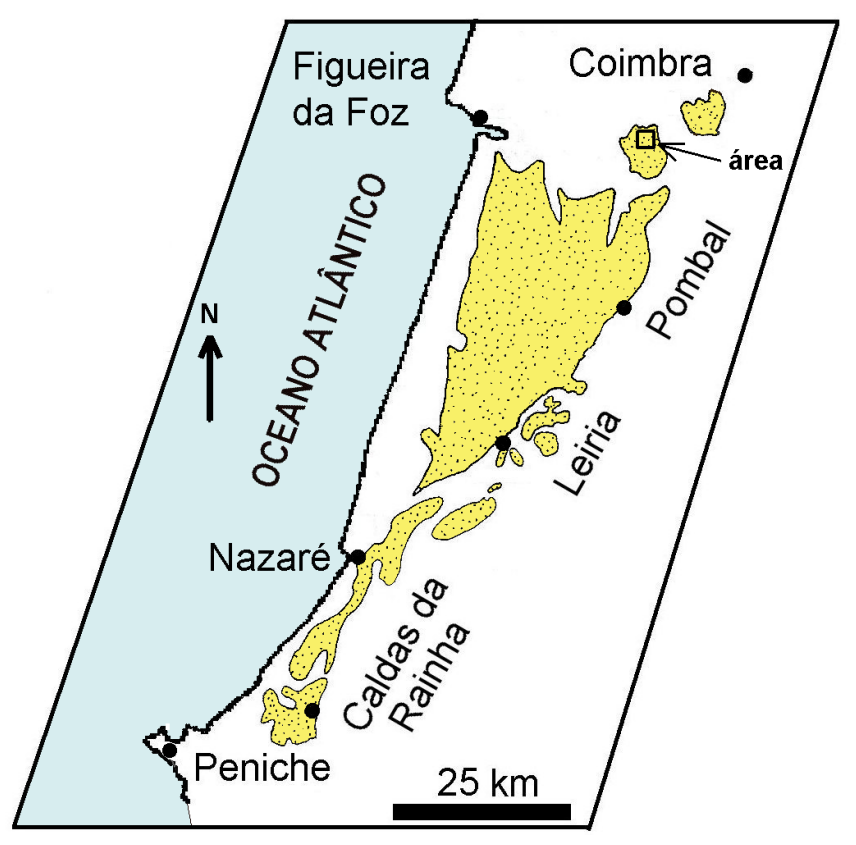

Fig. 1. Terrenos do neogénico no litoral ocidental de Portugal e localização da área do depósito (adapt. Reis, 1983).

\section{AMOSTRAGEM}

Neste primeiro estudo foi efetuada amostragem em três níveis: inferior, médio e superior (ou de topo) sendo as amostras designadas $P i, P m$ e $P t$, respetivamente. As amostras $P i$ e $P m$ foram colhidas na parte sudeste do depósito aproveitando uma pequena ra- vina que o destapa (figura 2). A amostra do nível superior foi colhida na parte central. O nível inferior tem uma tonalidade esverdeada enquanto o médio e o superior tem cor alaranjada (figura 3). Foram estudadas duas frações granulométricas, $<63 \mu \mathrm{m}$ e $<2$ $\mu \mathrm{m}$ nas quais se realizaram um conjunto de análises mineralógicas e tecnológicas. 


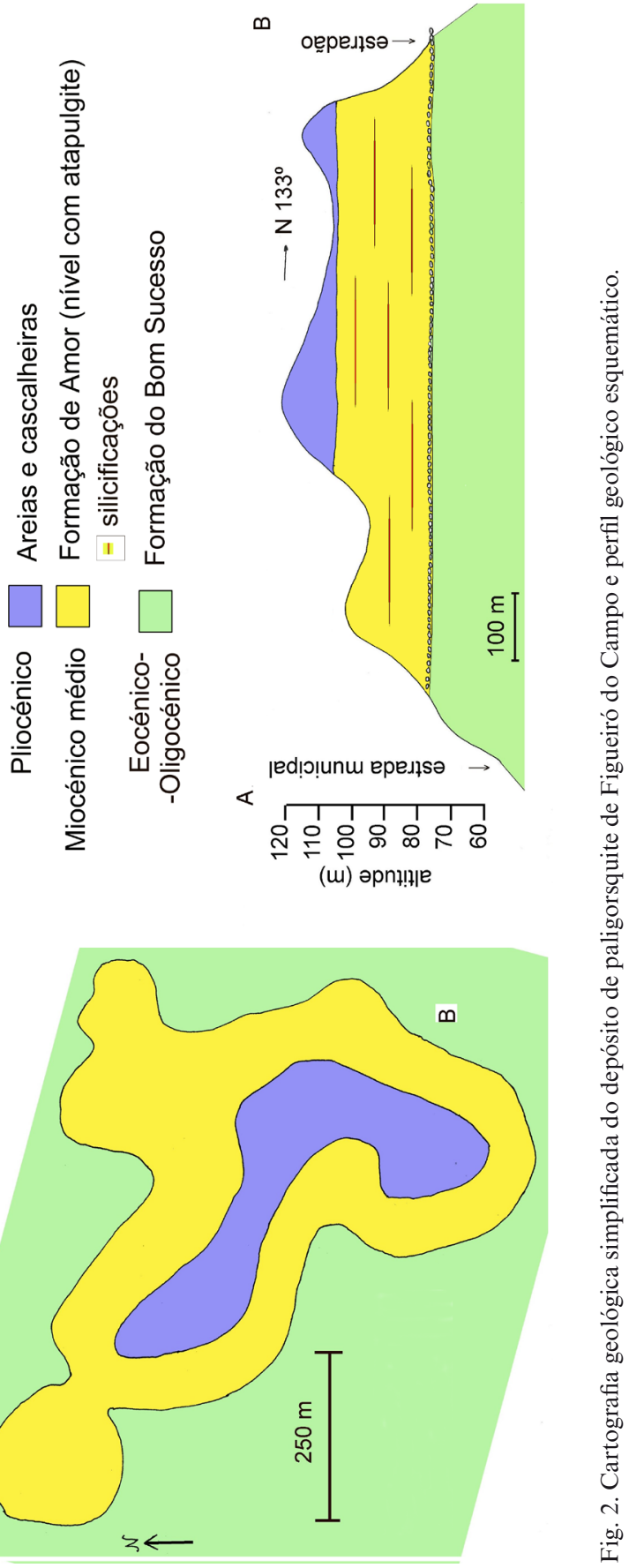




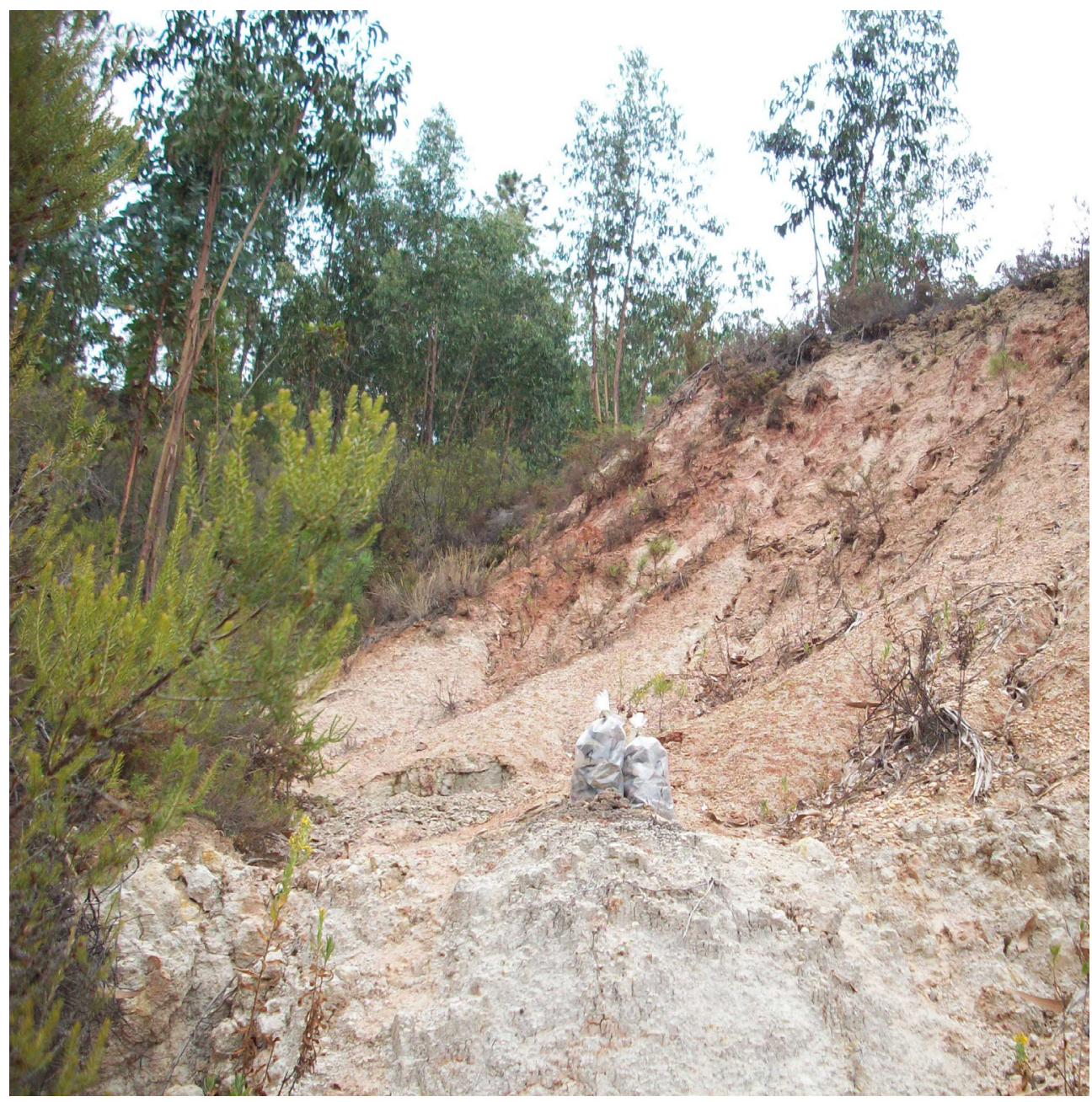

Fig. 3. Imagem do afloramento onde foram colhidas as amostras $\mathrm{Pb}$ e $\mathrm{Pm}$.

\section{MÉTODOS DE ESTUDO}

As amostras foram submetidas a difração de raios $X$ (DRX), fluorescência de raios $\mathrm{X}$ (FRX), microscopia eletrónica de varrimento $(\mathrm{MEV})$, medição de $\mathrm{pH}$, testes de absorção de água e de óleo, de abrasividade e de capacidade de troca catiónica.
Os DRX foram executados na amostra total e nas frações $<63 \mu \mathrm{m}$ e $<2 \mu \mathrm{m}$; nestas últimas foram executadas análises em amostras não orientadas e em amostras orientadas. Utilizou-se um difractómetro Philips X'Pert constituído por um gerador MPPC, um goniómetro $P W 3050$ e um microprocessador $P W 3040 / 60$ com registo em impressora. Nas 
análises químicas por FRX foi utilizado o equipamento Philips Automatic Bead Machine "Perl'X3". Nas observações no microscópio eletrónico de varrimento foi usado o equipamento Quanta 400FEG ESEM / EDAX Genesis X4M pertencente ao Centro de Materiais da Universidade do Porto. $\mathrm{O}$ pH foi medido no aparelho Crison-Hanna -HI 8014 calibrado com soluções padrão de valores de pH 4,0 e 7,2; nestas análises a relação amostra/água foi de 1:2,5. Nos testes de absorções de água e de óleo usou-se a norma ISO 787/51980. A abrasividade foi determinada num abrasivímetro Einlehner AT-1000 e para a capacidade de troca catiónica foi adotado o procedimento que utiliza acetato de amónio e que se encontra descrito em ALVES (2013).

\section{RESULTADOS}

\subsection{Granulometria}

As amostras estudadas correspondem a siltitos com uma percentagem de fração $>63$ $\mu \mathrm{m}$ variando entre $20 \%$ e $40 \%$. A fração $<4$ $\mu \mathrm{m}$ corresponde a $49-62 \%$ da rocha total (resultados obtidos a partir da análise com o equipamento Segigraph 6100).

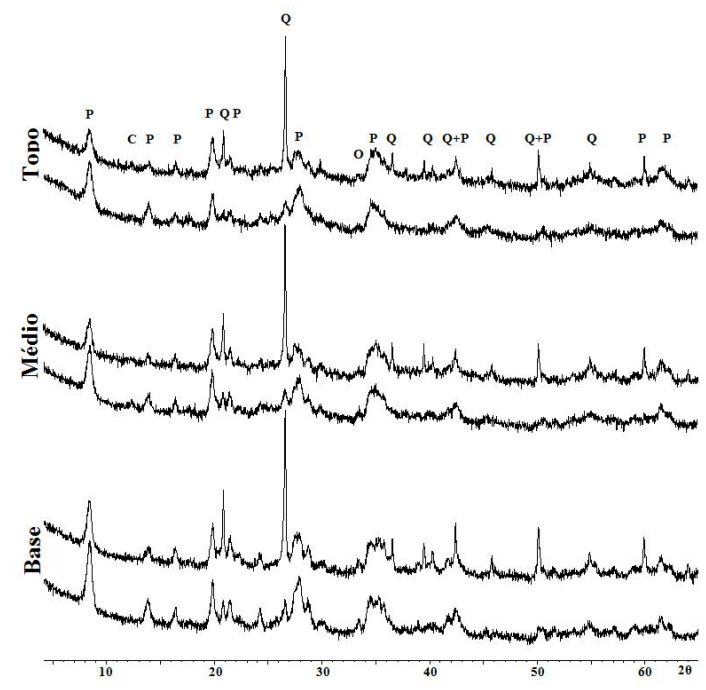

Fig. 4. Difractogramas correspondentes a amostras não orientadas das fracções $<63 \mu \mathrm{m}$ (espectros superiores) e $<2 \mu \mathrm{m}$ (espectros inferiores). P: paligorsquite, Q: quartzo, C: caulinite e O: óxidos de ferro (hematite e goethite).

\subsection{Difração de raios $X$ (DRX)}

Na figura 4 encontram-se representados os difratogramas das amostras estudadas, correspondentes às frações $<63 \mu \mathrm{m}$ e $<2 \mu \mathrm{m}$. A amostra total apresenta um DRX idênti- co embora com um pouco mais de quartzo e menos paligorsquite. Os resultados obtidos revelam uma clara predominância de paligorsquite e de quartzo, sendo esta última a impureza dominante. Tendo em conta a semiquantificação, indicada na tabela 1 , é 
possível concluir que em todas as amostras se verifica uma diminuição do teor em quartzo e um aumento do teor em paligorsquite da fração $<63 \mu \mathrm{m}$ para a $<2 \mu \mathrm{m}$. Os óxidos de ferro, representados pela hematite e pela goethite, ocorrem em pequena quanti- dade. Nenhum dos difratogramas exibe picos relativos a esmectite ou sepiolite (figura 5). As amostras $P m$ e $P t$ apresentam vestígios de caulinite, sendo que a maior quantidade ocorre na fração $<2 \mu \mathrm{m}$ da amostra $P t$ (tabela 1).
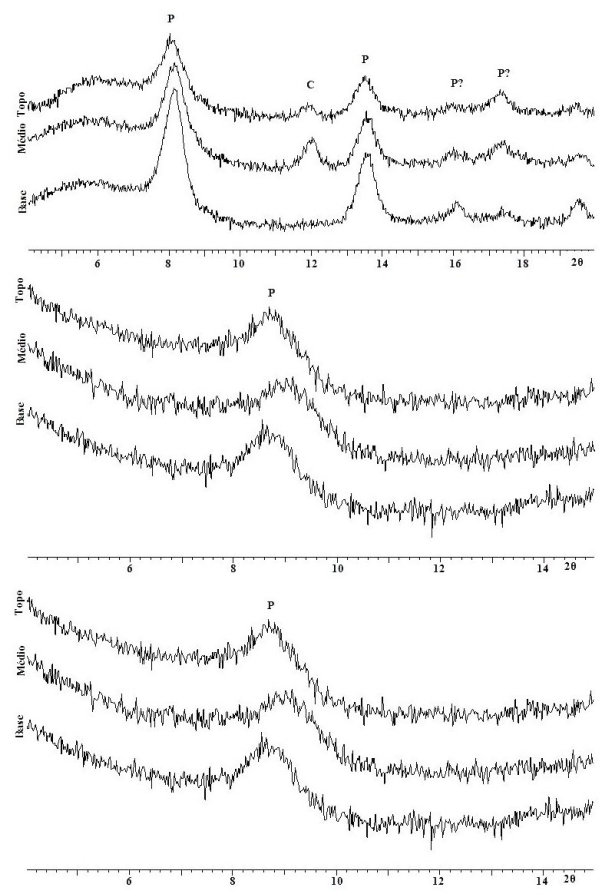

Fig. 5. Difractogramas de amostras orientadas correspondentes à fração $<2 \mu \mathrm{m}$.

A - Estado natural, em que P representa a paligorsquite e C a caulinite; B - Etilenoglicol; C - Após aquecimento a $500{ }^{\circ} \mathrm{C}$.

\begin{tabular}{|c|c|c|c|c|c|}
\hline & & $\begin{array}{l}\text { Paligorsquite } \\
(\%)\end{array}$ & $\begin{array}{c}\text { Quartzo } \\
(\%)\end{array}$ & $\begin{array}{c}\text { Óxidos de ferro } \\
\text { (\%) }\end{array}$ & $\begin{array}{c}\text { Caulinite } \\
(\%)\end{array}$ \\
\hline \multirow{2}{*}{$P t$} & $<63 \mu \mathrm{m}$ & 61 & 34 & 5 & $*$ \\
\hline & $<2 \mu \mathrm{m}$ & 89 & 8 & 3 & $*$ \\
\hline \multirow{2}{*}{$P m$} & $<63 \mu \mathrm{m}$ & 63 & 31 & 6 & $*$ \\
\hline & $<2 \mu \mathrm{m}$ & 89 & 7 & 4 & 3 \\
\hline \multirow{2}{*}{$P b$} & $<63 \mu \mathrm{m}$ & 70 & 26 & 4 & nd \\
\hline & $<2 \mu \mathrm{m}$ & 93 & 4 & 3 & nd \\
\hline
\end{tabular}

Tabela. 1. Semiquantificação dos minerais existentes nas amostras, em percentagem. P: paligorsquite, Q: quartzo, C: caulinite e O: óxidos de ferro (hematite e goethite). 


\subsection{Análise química}

Na tabela 2 encontram-se indicados os resultados das análises químicas obtidos por espectrometria de fluorescência de raios $\mathrm{X}$, dos elementos maiores correspondentes às três amostras e, para cada uma, às duas frações granulométricas $(<63 \mu \mathrm{m}$ e $<2 \mu \mathrm{m})$. Os teores de $\mathrm{SiO}_{2}, \mathrm{Al}_{2} \mathrm{O}_{3}, \mathrm{MgO}$ e Fe total são os de maior destaque. É importante salientar a diminuição dos teores em $\mathrm{SiO}_{2}$, e o aumento dos teores em $\mathrm{Al}_{2} \mathrm{O}_{3}, \mathrm{Fe}$ total e $\mathrm{MgO}$ (excetuando os teores de $\mathrm{MgO}$ da amostra $P t$ ) da fração $<63 \mu \mathrm{m}$ para a $<2$ $\mu \mathrm{m}$, o que indica uma diminuição do teor em quartzo e um aumento da paligorsquite. Por outro lado verifica-se que da base para o topo da coluna estratigráfica os teores de Fe total têm tendência para aumentar e o mesmo acontece com os de $\mathrm{Al}_{2} \mathrm{O}_{3}, \mathrm{TiO}_{2}$, $\mathrm{CaO}, \mathrm{Na}_{2} \mathrm{O}$ e $\mathrm{K}_{2} \mathrm{O}$. Os teores de $\mathrm{SiO}_{2}$ e de
MgO mostram uma tendência oposta, isto é, verifica-se uma diminuição daqueles teores à medida que se sobe na coluna estratigráfica, que se encontra relacionada com o aumento do teor em $\mathrm{Al}_{2} \mathrm{O}_{3}$. Na tabela 3 encontram-se indicadas as relações entre os elementos $\mathrm{Si}$, $\mathrm{Al}$ e Mg. As relações observadas vêm corroborar algumas observações decorrentes dos resultados das análises químicas dos elementos maiores. As relações $\mathrm{Si} / \mathrm{Al}$ e $\mathrm{Si} /$ $\mathrm{Mg}$ diminuem das frações $<63 \mu \mathrm{m}$ para as $<2 \mu \mathrm{m}$, devido à diminuição de sílica da fração $<63 \mu \mathrm{m}$ para a fração $<2 \mu \mathrm{m}$, sendo esta última mais rica em $\mathrm{Al}$ e $\mathrm{Mg}$ devido à presença de paligorsquite. Verifica-se, também, um aumento dos teores de $\mathrm{Al}$ em relação aos de $\mathrm{Mg}$ (a razão $\mathrm{Al} / \mathrm{Mg}$ aumenta) da base para o topo, o que indica que o $\mathrm{Al}$ poderá estar a substituir o Mg na estrutura (nomeadamente nas camadas octaédricas) da paligorsquite.

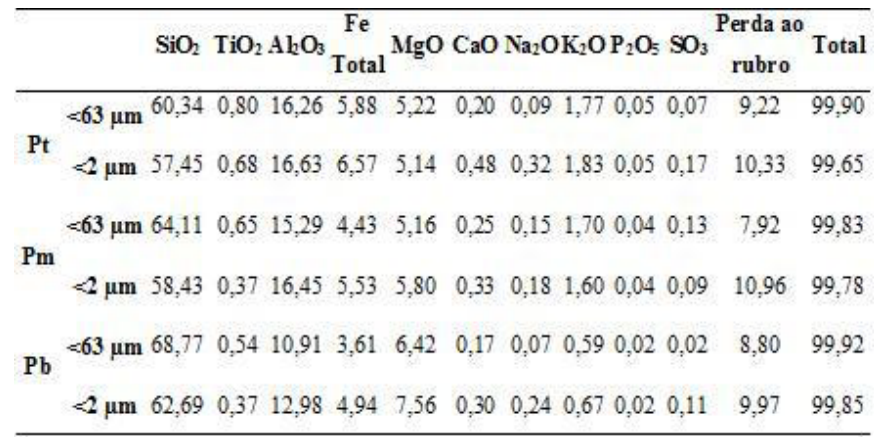

Tabela. 2. Resultados das análises químicas dos elementos maiores em percentagem de óxidos.

\subsection{Microscopia eletrónica de varrimento (MEV)}

As amostras foram analisadas no microscópio eletrónico com o objetivo de estudar a dimensão e a forma das partículas de paligorsquite. Na figura 6 mostram-se algumas das observações mais significativas obtidas por MEV. Foram medidas várias fibras, com os seguintes resultados: comprimento médio $=344 \mathrm{~nm}$ e valores no intervalo $[106,980] \mathrm{nm}$; largura média $=31 \mathrm{~nm}$, com valores no intervalo $[14,50] \mathrm{nm}$, sendo a distribuição a que se pode observar na 
figura 7. É possível observar e identificar na imagem A, alguns aglomerados e partículas fibrosas. Estes aglomerados são comuns e devem-se ao facto das partículas possuírem elevada capacidade de absorção, o que faz com que as partículas individualizadas se agreguem. Observam-se também algumas, embora poucas, partículas com cariz lamelar hexagonal, como acontece com a amostra $\mathrm{Pi}$, correspondente à imagem $\mathrm{B}$. Estas partículas correspondem a caulinite. Em todas as amostras foi também possível observar partículas de forma globular irregular, as quais poderão corresponder a quartzo Finalmente, não se observam diferenças nas características fibrosas das partículas de paligorsquite entre as amostras.
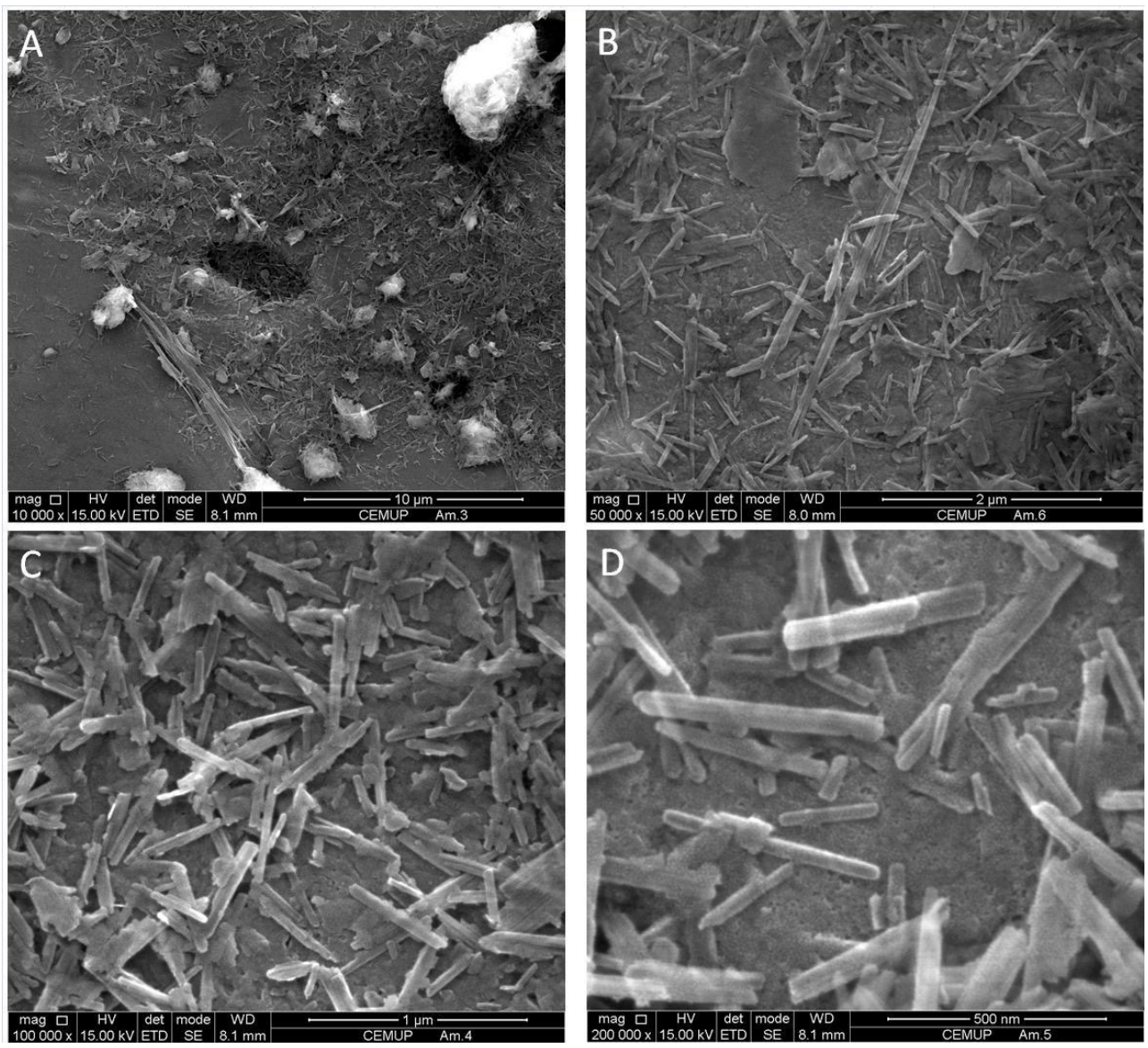

Fig. 6. Fotografias retiradas em MEV, onde se torna evidente o hábito fibroso das partículas. A - amostra Pt $<63 \mu \mathrm{m} ; \mathrm{B}-$ amostra $\mathrm{Pt}<2 \mu \mathrm{m} ; \mathrm{C}-$ amostra $\mathrm{Pi}<2 \mu \mathrm{m}$; D - amostra $\mathrm{Pb}<2 \mu \mathrm{m}$. 


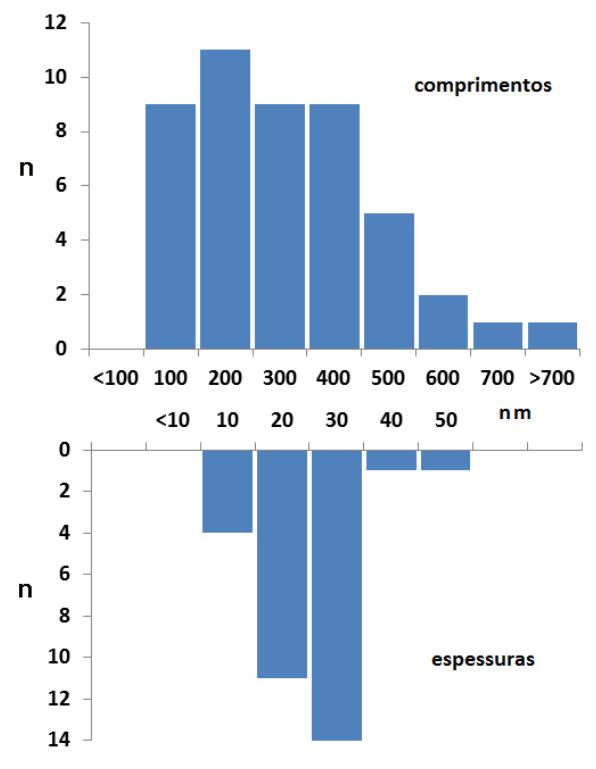

5.5. Propriedades tecnológicas

$\mathrm{Na}$ tabela 4 encontra-se indicado um conjunto de propriedades selecionadas que caracterizam a paligorsquite do ponto de vista tecnológico.

Os resultados mostram as diferenças marcantes entre as propriedades das frações $<63 \mu \mathrm{m}$ e $<2 \mu \mathrm{m}$ sobretudo em termos de abrasividade e de absorção de óleo e de água. A presença de quartzo constitui uma limitação clara do depósito em estudo o qual é responsável por maior abrasividade e menores valores de absorção de óleo e de água. Por outro lado, no que se refere à capacidade de troca catiónica, verifica-se uma tendência para aumentar à medida que se sobe na escala estratigráfica, se bem que, com exceção da amostra $P i$, não se note a influência da fração granulométrica, como seria de esperar. De facto, entre as frações granulométricas $<63 \mathrm{~mm}$ e $<2 \mathrm{~mm}$, não se detetam diferenças marcantes à exceção da amostra $P i$, o que
Fig. 7.Distribuição dos comprimentos e espessuras de fibras de paligorsquite medidas no MEV.

poderá relacionar-se com os baixos valores daquela propriedade por comparação com os valores considerados habituais para a paligorsquite, que variam entre $20 \mathrm{meq}$ e $50 \mathrm{meq}$ por $100 \mathrm{~g}$ (VELHO, 2005).

\section{DISCUSSÃO}

\subsection{Análise tecnológica}

Os resultados de DRX traduzem uma diminuição do teor em paligorsquite da base para o topo em ambas as frações bem como um aumento do teor em quartzo e em caulinite. A mineralogia da fração $<2 \mu \mathrm{m}$ é favorável à utilização da argila na maioria das aplicações, mas o mesmo não se passa na fração $<63 \mu \mathrm{m}$ devido aos teores de quartzo elevados.

Os teores em $\mathrm{MgO}$ diminuem da base para o topo em consonância com a diminuição do teor em paligorsquite, no entanto as mesmas análises mostram uma tendência na 
diminuição do teor em sílica da base para o topo facto que não se coaduna com o aumento do teor em quartzo no sentido ascendente. Esta discrepância deverá ser resolvida com o aumento do número de amostras analisadas.

Os resultados preliminares até agora obtidos mostram que o principal contaminante da paligorsquite é o quartzo que a acompanha até à fração argilosa $(<2 \mu \mathrm{m})$ se bem que aqui em quantidade diminuta. O quartzo é responsável por retirar à paligorsquite características como a capacidade de troca catiónica e propriedades de absorção, dois atributos muito importantes em termos de potencial de aplicações.

Apesar da presença ubíqua de quartzo, os dados obtidos a partir dos ensaios de absorção de água e de óleo são favoráveis e assemelham-se com os requisitos para as aplicações típicas da paligorsquite (HARBEN, 2002; LUZ, 2008). Os dados de abrasividade correspondentes à fração $<2 \mu \mathrm{m}$, para todas as amostras, são positivos e refletem a diminuição do teor em quartzo, no entanto, para a fração $<63 \mu \mathrm{m}$ os dados de abrasividade são elevados e constituem uma limitação do depósito de paligorsquite.

No entanto os valores da capacidade de troca catiónica são insuficientes, devendo ser melhorados, tendo em vista o uso industrial desta matéria-prima. Para tal será decisivo diminuir fortemente o teor em quartzo através de processos de beneficiação como o hidrociclone juntamente com a peneiração e ativar as amostras de paligorsquite com ácido. Ensaios de ativação ácida recentemente realizados (ALVES, 2013; VELHO et al., 2014) sobre amostras do mesmo material, mostraram um aumento significativo da capacidade de absorção, e ao mesmo tempo tornaram o material mais claro

\subsection{Análise sobre a génese}

Observa-se frequentemente nos sedimentos onde a paligorsquite ocorre, a existência conjunta de esmectite, para além de ilite, ocasionalmente sepiolite e em alguns casos, de caulinite. A formação da paligorsquite é atribuída a neoformação ou a transformação a partir de outro mineral (em geral esmectite), requerendo um meio aquoso com $\mathrm{pH}<8,5$, baixa pressão de $\mathrm{CO}_{2}$ e salinidade alcalina moderada.

Em termos gerais, os principais fatores que influenciam a ocorrência de paligorsquite são (JONES E CONKO, 2011): 1- Material de origem: rochas graníticas a ultramáficas, bem como sedimentos lacustres; 2- Clima: árido a semiárido, com chuvas restritas, dependendo das temperaturas regionais (temperado a quente); 3- Parâmetros físico-químicos: o $\mathrm{pH}$ ideal para a formação de paligorsquite é entre 6 e 8,5. Águas com $\mathrm{pH}>9,5$, elevada alcalinidade salina, elevada pressão em $\mathrm{CO}_{2}$ e elevadas razões $(\mathrm{Mg}+\mathrm{Fe}) / \mathrm{Si}$ são fatores desfavoráveis à formação desta argila (GUGGENHEIM E KREKELER, 2011).

A montmorilonite é proposta como mineral aluminoso precursor. As reações em solução aquosa entre silicatos que contêm alumina, sílica e $\mathrm{Mg}^{2+}$ são uma condição importante na génese da paligorsquite (WEAVER e BECK, 1977; GALÁN e POZO, 2011). A velocidade da dissolução aquosa de uma fase precursora (por exemplo, esmectite) pode ser aumentada de modo a que a nova fase (paligorsquite) possa ser gerada em pouco tempo.

Em ambientes de sedimentação continental, a existência de um substrato lítico rico em magnésio, alumínio e silício é um fator favorável à formação de paligorsquite. 
No entanto a entrada de sílica no sistema pode resultar de qualquer rocha magmática, metamórfica ou sedimentar, que possua o quimismo favorável. Neste aspeto, basaltos ou gabros olivínicos, dolomitos e argilitos esmectiticos constituem as rochas mais favoráveis a fornecerem o magnésio (em particular) para a formação de paligorsquite. Estratigraficamente o Cenozoico envolvente da região é caracterizado pela predominância de sedimentos siliciclásticos de ambientes fluviais em clima temperado. Alguns quilómetros a sudeste do depósito ocorrem dolomitos e calcários dolomíticos de idade jurássica. A presença de paligorsquite implica uma mudança nas características ambientais com aumento da temperatura e alcalinidade das águas, traduzida por um quimismo mais rico em alumínio e, sobretudo, em magnésio. Esta mudança climática durante o miocénico médio tem sido reportada a nível mundial traduzida por um aumento da aridez (MILLER e FAIRBANKS, 1983). Na área de Figueiró do Campo, REIS (1983) observou amostras com paligorsquite e esmectite para além de amostras com paligorsquite mas sem esmectite, bem como pequenas quantidades de caulinite na metade superior do corte analisado.

Nas amostras deste estudo detetou-se, para além da paligorsquite, pequenas quantidades de caulinite (em algumas amostras) mas não se observou esmectite.

\section{CONCLUSÕES}

Dos estudos realizados até ao momento podemos concluir o seguinte:

1. Mineralogicamente, os principais aspetos a referir são a presença contínua de quartzo e de paligorsquite da base ao topo do depósito. O quartzo é responsável pelo menor desempenho ao nível da absorção e de capacidade de troca catiónica. A presença do contaminante ferro é responsável pela coloração amarelada de parte do depósito.

2. A qualidade geral do litótipo obriga à necessidade de processos de beneficiação para a remoção de parte do quartzo, principal contaminante, através, por exemplo do uso de um hidrociclone, juntamente com peneiração, seguida de ativação com ácido de modo a incrementar as propriedades de absorção e de capacidade de troca catiónica.

3. O estudo confirmou o potencial industrial do depósito, após beneficiação, pelo menos nas aplicações tradicionais, aproveitando as suas características absorventes.

4. O mecanismo genético responsável pela formação de paligorsquite envolve uma mudança radical no ambiente caracterizado por condições mais alcalinas, com $\mathrm{pH}$ mais elevado, traduzido por um ambiente quente e seco favorável à presença de águas com elevado teor em magnésio, silício, alumínio e ferro.

\section{REFERÊNCIAS}

ALVES, W., 2013. Caracterização da paligorsquite de Figueiró do Campo. Tese de Mestrado. Universidade de Aveiro.

CUNHA, P. P. e REIS, R. P., 1989. Principais ocorrências de paligorsquite, em depósitos de idade cretácica superior e terciária, em Portugal Central. $1^{\circ}$ Reunião Luso-Espanhola de Argilas, p 22.

CUNHA P. P, REIS R. P., 1992. Establishment of unconformity-bounded sequences in the Cenozoic record of the western Iberian margin and synthesis of 
the tectonic and sedimentary evolution in central Portugal during Neogene. Abstracts of the First Congress RCANS, Lisboa, pp 33-35.

DIAS, I., 1998. Caracterização Mineralógica e Tecnológica de Argilas Especiais de Bacias Terciárias Portuguesas. Tese de doutoramento. Faculdade de Ciências da Universidade de Lisboa.

GALÁN, E. e POZO, M., 2011. Palygorskite and sepiolite deposits in continental environments. Description, genetic patterns and sedimentary settings. Em Developments in Palygorskite-Sepiolite Research: A New Outlook on These Nanomaterials, E. Galán, A. Singer (editores), Elsevier. Developments of Clay Science. p. 125-166.

GALOPIM DE CARVALHO, A., 1968. Contribuição para o Conhecimento Geológico da Bacia Terciária do Tejo. Serviços Geológicos de Portugal, Memória $\mathrm{n}^{\circ} 15$ (Nova Série), Lisboa, 210p.

GUGGENHEIM, S. e KREKELER, M., 2011. The structure and microtextures of the palygorskite-sepiolite group minerals. Developments in palygorskite-sepiolite research. A new outlook on these nanomaterials. Editado por Galan, E. e Singer, A. (editors). Elsevier. Developments in Clay Science 3, pp. 3-65.

HARBEN, P., 2002. The industrial minerals Handybook: A guide to markets, specifications and prices. $4^{\text {th }}$ edition. Metal Bulletin plc, London.

HEIVILIN., H. M., 1994. Hormites: Palygorskite (Attapulgite) and Sepiolite, in Industrial minerals and rocks, Littleton, Colorado: Society for Mining, Metallurgy and Exploration. p. 249-254.
JONES, B. e CONKO, K., 2011. Environmental influences on the occurrences of sepiolite and palygorskite: A brief review. Em Developments in PalygorskiteSepiolite Research: A New Outlook on These Nanomaterials, E. Galán, A. Singer(editores). Elsevier. Developments of Clay Science. p. 69-81.

LUZ, A. e ALMEIDA, S., 2008. Argila - Atapulgita e Sepiolita. Em Rochas e Minerais Industriais: Usos e Especificações. Parte 2 - Rochas e Minerais Industriais: Usos e Especificações. CETEM Rio de Janeiro, p. 223-238.

MILLER, K. e FAIRBANKS, R., 1983. Evidence for Oligocene-Middle Miocene abyssal circulation changes in the western North Atlantic. Nature, 306 (5940), p. 250-253.

PAIS, J., CUNHA, P. P., LEGOINHA, P., 2010. Litostratigrafia do Cenozóico de Portugal. Em Neiva, J.M. et al., (Editores). Ciências Geológicas e Investigação. V. I, 365-376.

REIS, R. P., 1983. A sedimentologia de depósitos continentais. Dois exemplos do Cretácico Superior-Miocénico de Portugal. Tese de doutoramento. Universidade de Coimbra.

VELHO, J., 2005. Mineralogia Industrial. Princípios e Aplicações. Edições Lidel, Lisboa, $600 \mathrm{p}$.

VELHO, J., MOURA, A. e ALVES, W., 2014. Activação ácida em sedimentos paligorsquiticos da região de Soure. (trabalho submetido a congresso).

WEAVER, C., e BECK, K., 1977. Miocene of the S.E. United States: a model for chemical sedimentation in a perimarine environment. Development in Sedimentology, 22: p. 234. 